\title{
Influência da densificação da sílica ativa nas propriedades de materiais cimentícios
}

\author{
Influence of densification of silica fume on the properties \\ of cementitious materials
}

\begin{abstract}
Yuri Sotero Bomfim Fraga
Lucas Martins Gontijo Soares

Valdirene Maria Silva Capuzzo

\section{Resumo}

A

sílica ativa é comercializada na forma densificada (SD), quando submetida ao beneficiamento por aglomeração das partículas para reduzir seu volume durante o transporte, ou não densificada (SND), quando esse processo não ocorre. Dessa forma, esta pesquisa tem como objetivo avaliar se a densificação da sílica ativa pode influenciar nas propriedades dos materiais cimentícios. Para isso, foi verificado o efeito da substituição de $10 \%$ do cimento Portland CP V-ARI pelas sílicas densificada e não densificada. Foi realizado o ensaio de resistência à compressão dos microconcretos aos 1, 3, 7, 14 e 28 dias. Para explicar o comportamento mecânico dos microconcretos, foram realizados os ensaios de difração de raios X (DRX) e microscopia eletrônica de varredura (MEV) com espectroscopia por dispersão de energia (EDS) nas pastas de cimento. Devido ao menor tamanho das partículas e à maior superfície específica da SND em comparação com a SD, houve alteração na microestrutura das pastas de cimento. A SND proporcionou maior consumo do hidróxido de cálcio $(\mathrm{CH})$ e menor relação cálcio/sílica $(\mathrm{Ca} / \mathrm{Si})$ da matriz cimentícia, resultando em maior resistência à compressão do microconcreto com SND em relação à SD. Dessa forma, conclui-se que a SND é mais eficaz quando utilizada na produção de materiais cimentícios.
\end{abstract}

Palavras-chave: Cimento Portland. Materiais pozolânicos. Microestrutura. Sílica ativa densificada e sílica ativa não densificada.

${ }^{1}$ Yuri Sotero Bomfim Fraga UUniversidade de Brasília Brasília - DF - Brasil

${ }^{2}$ Lucas Martins Gontijo Soares ZUniversidade de Brasília Brasília - DF - Brasil

${ }^{3}$ Valdirene Maria Silva Capuzzo ${ }^{3}$ Universidade de Brasília Brasília - DF - Brasil

Recebido em 05/02/20 Aceito em 22/11/20

\section{Abstract}

The silica fume is commercially available in densified form (DS) when subjected to particle agglomeration processing to reduce its volume during transport, or undensified (US) when that process does not occur. The purpose of this study is to evaluate whether the densification of silica fume can influence the properties of cementitious materials. With that purpose, the effect of replacing $10 \%$ of Portland cement CP V-ARI by US and DS was investigated. Compressive strength tests were performed on the microconcrete at 1,3, 7, 14 and 28 days. To explain the mechanical behaviour of the microconcrete, X-ray diffraction (XRD) and scanning electron microscopy (SEM) with energy dispersion spectroscopy (EDS) tests were performed on the cement pastes. Due the smaller particle size and the higher specific surface of the US compared to the DS, there was a change in the microstructure of the cement pastes. The US provided a higher consumption of calcium hydroxide $(\mathrm{CH})$ and a lower calcium/silica $(\mathrm{Ca} / \mathrm{Si})$ ratio in the cementitious matrix, resulting in a higher compressive strength in the microconcrete with US compared to that with DS. The study concludes that US is more effective when used in the production of cementitious materials.

Keywords: Portland cement. Pozzolanic materials. Microstructure. Densified silica fume and undensified silica fume. 


\section{Introdução}

Com o avanço tecnológico, exige-se cada vez mais que os materiais utilizados na indústria da construção civil possuam propriedades adequadas para determinada finalidade. $\mathrm{O}$ concreto de alto desempenho (CAD), por exemplo, possui elevado desempenho mecânico e durabilidade devido à sua microestrutura densa. Esse tipo de concreto é utilizado em estruturas com elevado índice de esbeltez, com grandes vãos e que exigem elevada durabilidade (CITEK et al., 2016).

A melhoria das propriedades dos materiais cimentícios ocorre com a redução da relação água/aglomerante e com a utilização de materiais cimentícios suplementares (MCS) altamente reativos. Um dos principais MCS altamente reativos utilizados na construção civil para a produção do CAD é a sílica ativa (SA) devido ao seu elevado grau de amorfismo, finura e superfície específica (FRAGA et al., 2020; GESOGLU et al., 2016; ZHANG; ZHANG; YAN, 2016b).

A SA é um resíduo oriundo da produção de ligas de ferro-silício e silício metálico. O seu elevado teor de $\mathrm{SiO}_{2}$ amorfo, geralmente superior a $85 \%$, e a sua elevada finura são as principais razões de sua alta atividade pozolânica (SIDDIQUE, 2011). A reação pozolânica é dada pela interação da sílica amorfa com o hidróxido de cálcio $(\mathrm{CH})$ proveniente da hidratação do cimento Portland, consumindo o $\mathrm{CH}$ para a formação de silicato de cálcio hidratado (C-S-H) adicional. Além disso, devido à sua elevada finura, a SA atua no efeito fíler criando pontos de nucleação, o que resulta no refinamento da estrutura porosa de pastas, argamassas e concretos (AÏTCIN, 2000; JUENGER; SIDDIQUE, 2015; LOTHENBACH; SCRIVENER; HOOTON, 2011; OERTEL et al., 2014; SIDDIQUE, 2011; ZHANG; ZHANG; YAN, 2016b).

Esse material pode ser classificado como não densificado (SND) ou densificado (SD). A SND é a sílica ativa em seu estado natural, com densidade aparente no estado solto entre $150 \mathrm{~kg} / \mathrm{m}$ e $350 \mathrm{~kg} / \mathrm{m}$ (ABNT, 2012). A SD é a sílica ativa submetida a beneficiamento por aglomeração de partículas, resultando em densidade entre $350 \mathrm{~kg} / \mathrm{m}$ e $700 \mathrm{~kg} / \mathrm{m}$. Esse beneficiamento para aumentar a sua densidade geralmente é realizado para facilitar o transporte e o manuseio da SA (ABNT, 2012; ZHANG; ZHANG; YAN, 2016b).

As partículas de SND possuem diâmetros menores que $10 \mu \mathrm{m}$, enquanto a SD pode apresentar aglomerados com tamanhos de até centenas de micrômetros. O tamanho e a área superficial dessas partículas influenciam diretamente na atividade pozolânica da SA. Dessa forma, quanto menor o seu tamanho e maior a sua superfície específica, maior é a reatividade da SA (BAJJA et al., 2017; BARBHUIYA; CHOW; DAS, 2014; FRAGA et al., 2020; ZHANG; ZHANG; YAN, 2016a). Constata-se que, em geral, a SND possui menores partículas que a SD e consequentemente a SND possui maior reatividade.

$\mathrm{Na}$ literatura, a influência do tipo de SA foi estudada em pastas, argamassas e concretos. Em um dos estudos, foram pesquisados os teores de $5 \%$ e $10 \%$ de SA e as relações água/aglomerante de 0,24 e 0,29. Foi observado que, inicialmente, a resistência à compressão dos materiais cimentícios sem a SA foi maior, porém, com o decorrer da hidratação, as pastas, as argamassas e os concretos com SA resultaram em maior resistência à compressão que os materiais sem SA. Os materiais cimentícios produzidos com SND resultaram em maior resistência que os com SD. Houve maior consumo de $\mathrm{CH}$ para a formação de $\mathrm{C}-\mathrm{S}-\mathrm{H}$ dos materiais com SND em relação aos com SD. Isso foi associado ao menor tamanho das partículas da SND em relação à $\mathrm{SD}$, que resultou na maior reatividade da SND e na maior efetividade do efeito fíler (ZHANG; ZHANG; YAN, 2016a).

A SA pode sofrer duas reações distintas: reagir com $\mathrm{CH}$ formando $\mathrm{C}-\mathrm{S}-\mathrm{H}$ com baixo teor de cálcio ou reagir com os álcalis da pasta de cimento formando gel de reação álcali-sílica (DIAMOND; SAHU; THAULOW, 2004). A primeira reação gera um produto estável, enquanto a segunda produz um produto com instabilidade volumétrica. Além disso, alguns estudos mostram que a utilização da SA em conjunto com o cimento Portland resulta em alterações na microestrutura dos materiais cimentícios, promovendo a redução da relação Ca/Si do C-S-H (ANDRADE et al., 2019; DIAMOND; SAHU; THAULOW, 2004; FRAGA et al., 2020; GUO et al., 2019; RICHARDSON, 2000; ROSSEN; LOTHENBACH; SCRIVENER, 2015).

Poucos estudos abordam a diferença que os dois tipos de SA exercem no desempenho mecânico e na microestrutura de materiais cimentícios. Dessa forma, o objetivo deste artigo é avaliar a resistência à compressão de microconcretos com substituição parcial do cimento Portland pela sílica ativa densificada ou não densificada, bem como o efeito desses materiais no consumo do hidróxido de cálcio e na relação $\mathrm{Ca} / \mathrm{Si}$ de pastas de cimento.

230 Fraga, Y. S. B.; Soares, L. M. G.; Capuzzo, V. M. S. 


\section{Materiais e métodos}

\section{Materiais}

Para a preparação dos microconcretos e das pastas de cimento desta pesquisa, foram utilizados os seguintes materiais: cimento Portland CP V-ARI devido ao baixo teor de adições em sua composição, atendendo aos requisitos da NBR 16697 (ABNT, 2018); sílica ativa densificada com densidade aparente no estado solto igual a $595 \mathrm{~kg} / \mathrm{m}$, atendendo aos requisitos da NBR 13956-1 (ABNT, 2012); sílica ativa não densificada com densidade aparente no estado solto igual a $315 \mathrm{~kg} / \mathrm{m}$, atendendo aos requisitos da NBR 13956-1 (ABNT, 2012); areia natural quartzosa oriunda da região do Distrito Federal/Brasil nas granulometrias fina, média fina, média grossa e grossa, de acordo com a granulometria estabelecida na NBR 7215 (ABNT, 1997); aditivo superplastificante (SP) à base de policarboxilato Glenium 51 da Basf; e água da Companhia de Saneamento Ambiental do Distrito Federal (Caesb).

Na Tabela 1 é mostrada a composição química do cimento Portland, da sílica ativa densificada e da sílica ativa não densificada. $\mathrm{O}$ equipamento utilizado nesse ensaio foi o espectrômetro de fluorescência de raios $\mathrm{X}$ por energia dispersiva (XRF/EDX), modelo EDX-720HS da Shimadzu. Observa-se que os dois tipos de sílica ativa utilizados atendem aos requisitos da NBR 13956-1 (ABNT, 2012) quanto ao teor de $\mathrm{SiO}_{2}$, que deve ser superior a $85 \%$ para a SA ser utilizada em pastas, argamassas e concretos.

\section{Metodologia}

\section{Microscopia eletrônica de varredura da sílica ativa}

A morfologia dos dois tipos de sílica ativa utilizados na pesquisa foi observada a partir da utilização do microscópio eletrônico de varredura (MEV) modelo Jeol Quanta 450, operado em vácuo, com tensão de aceleração de elétrons (HV) de $5 \mathrm{kV}$. As micrografias foram obtidas por meio de elétrons secundários com detector vCD.

\section{Resistência à compressão axial dos microconcretos}

Para avaliar a resistência à compressão axial, foram preparados três diferentes microconcretos, sendo um traço referência (REF) somente com cimento Portland e os outros dois traços substituindo, em massa, 10\% do cimento Portland por sílica ativa densificada ou não densificada. A relação água/aglomerante foi fixada em 0,48. A quantidade de aditivo superplastificante foi ajustada para que todos os microconcretos resultassem na consistência de $150 \pm 10 \mathrm{~mm}$ no ensaio da mesa de consistência. Inicialmente, foi necessário $0,30 \%$ de aditivo superplastificante em relação à massa de cimento Portland para o microconcreto REF atingir o espalhamento mencionado. Esse mesmo teor foi utilizado para os microconcretos SD e SND para estimar, por tentativa e erro, a quantidade de aditivo superplastificante necessária para conseguir a consistência desejada, obtendo para esse teor $(0,30 \%)$ espalhamentos dentro da tolerância estipulada inicialmente $(150 \pm 10 \mathrm{~mm})$. Apesar de o teor de aditivo superplastificante ser o mesmo entre os microconcretos, foi observado que os microconcretos com sílica ativa (SD ou SND) resultaram em espalhamentos semelhantes entre si e menores que o microconcreto REF, corroborando com os resultados da literatura, que mostram redução da fluidez quando a sílica ativa é incorporada em compósitos cimentícios devido à sua elevada superfície específica (ANDRADE et al., 2019; CHEN; DENG; LI, 2016; FRAGA et al., 2020). A consistência medida através do espalhamento na mesa de consistência e a quantidade de materiais utilizados para a preparação dos corpos de prova cilíndricos com $50 \mathrm{~mm}$ de diâmetro x $100 \mathrm{~mm}$ de altura de microconcretos são mostradas na Tabela 2.

A mistura dos microconcretos foi realizada em um misturador planetário e seguiu os procedimentos da NBR 7215 (ABNT, 1997). Em uma cuba de aço inox com volume de 5 1, inicialmente foram adicionados a água e o aditivo superplastificante. Depois, foram adicionados a sílica ativa (SD ou SND) e o CP V-ARI, sendo realizada a mistura por $30 \mathrm{~s}$ em velocidade baixa (140 rpm em torno do eixo). Sem parar o processo de mistura, foi incorporado o agregado miúdo, com todas as faixas granulométricas previamente misturadas, durante $30 \mathrm{~s}$. Imediatamente após adicionar todo o agregado miúdo, foi aumentada a velocidade do misturador planetário para a velocidade alta $(285 \mathrm{rpm}$ em torno do eixo) por $30 \mathrm{~s}$. Depois, o misturador foi desligado por 1 min e $30 \mathrm{~s}$, sendo realizada a raspagem do material aderido às paredes da cuba durante os primeiros $15 \mathrm{~s}$. Após esse intervalo, o misturador foi ligado novamente na velocidade alta por mais 1 min. 
Tabela 1 - Composição química do cimento Portland, da sílica ativa densificada e da sílica ativa não densificada

\begin{tabular}{|c|c|c|c|}
\hline Composição Química & Cimento Portland & SD & SND \\
\hline $\mathrm{CaO}(\%)$ & 73,98 & 2,57 & 3,62 \\
\hline $\mathrm{SiO}_{2}(\%)$ & 9,91 & 91,70 & 91,41 \\
\hline $\mathrm{Fe}_{2} \mathrm{O}_{3}(\%)$ & 4,34 & 0,36 & 0,46 \\
\hline $\mathrm{SO}_{3}(\%)$ & 3,72 & 0,13 & 0,21 \\
\hline $\operatorname{MgO}(\%)$ & 2,93 & - & - \\
\hline $\mathrm{Al}_{2} \mathrm{O}_{3}(\%)$ & 2,49 & 1,58 & 2,02 \\
\hline $\mathrm{K}_{2} \mathrm{O}(\%)$ & 1,64 & 3,38 & 2,13 \\
\hline $\mathrm{SrO}(\%)$ & 0,59 & 0,01 & 0,01 \\
\hline $\mathrm{TiO}_{2}(\%)$ & 0,31 & - & - \\
\hline $\mathrm{MnO}_{2}(\%)$ & 0,09 & 0,20 & 0,11 \\
\hline $\mathrm{CuO}(\%)$ & 0,02 & 0,03 & - \\
\hline $\mathrm{RbO}_{2}(\%)$ & - & 0,02 & - \\
\hline $\mathrm{ZnO}(\%)$ & - & 0,02 & 0,04 \\
\hline
\end{tabular}

Tabela 2 - Quantidade de materiais utilizados em cada etapa de mistura para moldagem dos corpos de prova cilíndricos com $50 \mathrm{~mm}$ de diâmetro $\times 100 \mathrm{~mm}$ de altura de microconcretos e espalhamento dos microconcretos na mesa de consistência

\begin{tabular}{|c|c|c|c|c|c|c|c|c|c|c|}
\hline \multirow{3}{*}{$\begin{array}{l}\text { Microcon- } \\
\text { creto }\end{array}$} & \multicolumn{9}{|c|}{ Material } & \multirow{3}{*}{$\begin{array}{c}\text { Espalha- } \\
\text { mento } \\
\text { (mm) }\end{array}$} \\
\hline & \multirow{2}{*}{$\begin{array}{c}\text { CP V- } \\
\text { ARI (g) }\end{array}$} & \multirow{2}{*}{$\begin{array}{l}\text { Sílica } \\
\text { ativa } \\
(\mathbf{g})\end{array}$} & \multicolumn{4}{|c|}{ Areia (g) } & \multicolumn{2}{|c|}{$\begin{array}{l}\text { Superplastifi- } \\
\text { cante }\end{array}$} & \multirow{2}{*}{$\begin{array}{c}\text { Água } \\
\text { (g) }\end{array}$} & \\
\hline & & & Fina & $\begin{array}{c}\text { Média } \\
\text { fina }\end{array}$ & $\begin{array}{l}\text { Média } \\
\text { grossa }\end{array}$ & $\begin{array}{c}\text { Gros- } \\
\text { sa }\end{array}$ & (g) & $(\%)$ & & \\
\hline$\overline{\mathrm{REF}}$ & 624,0 & - & 468 & 468 & 468 & 468 & 1,87 & 0,30 & 300 & 154,1 \\
\hline $\mathrm{SD}$ & 561,6 & 62,4 & 468 & 468 & 468 & 468 & 1,87 & 0,30 & 300 & 147,9 \\
\hline SND & 561,6 & 62,4 & 468 & 468 & 468 & 468 & 1,87 & 0,30 & 300 & 147,8 \\
\hline
\end{tabular}

Após o término da mistura, foi realizado o ensaio da mesa de consistência para verificar se o espalhamento atendia ao intervalo de $150 \pm 10 \mathrm{~mm}$, conforme mencionado anteriormente. Foram moldados 20 corpos de prova cilíndricos com $50 \mathrm{~mm}$ de diâmetro x $100 \mathrm{~mm}$ de altura para cada traço de microconcreto. Após a moldagem, os corpos de prova foram colocados em câmara úmida por $24 \mathrm{~h}$, com a superfície protegida para não alterar a relação água/aglomerante, e depois desmoldados e postos em cura submersa em água saturada de cal até a idade de verificação da resistência à compressão. Aos 1, 3, 7, 14 e 28 dias de hidratação, os corpos de prova foram retificados e rompidos em uma máquina universal para ensaios mecânicos.

Com os resultados de resistência à compressão dos microconcretos aos 1, 3, 7, 14 e 28 dias de hidratação, foi realizada uma análise de variância (one-way ANOVA) para verificar se houve diferença significativa na resistência à compressão entre os três microconcretos avaliados. Depois, foi realizado o teste de Duncan para agrupar os resultados de resistência à compressão em grupos homogêneos, levando em consideração a média e o desvio padrão da resistência à compressão axial com base em $95 \%$ de confiança.

\section{Ensaios microestruturais nas pastas de cimento}

Foram realizados os ensaios de difração de raios X (DRX) e microscopia eletrônica de varredura com espectroscopia por dispersão de energia (EDS) nas pastas de cimento Portland. Para verificar exatamente o comportamento da pasta presente no microconcreto, foi utilizada a mesma proporção de materiais que os microconcretos, exceto o agregado miúdo, que não foi empregado. A quantidade de materiais necessários para moldagem de dois corpos de prova cilíndricos com $50 \mathrm{~mm}$ de diâmetro x $100 \mathrm{~mm}$ de altura de pastas de cimento é mostrada na Tabela 3. 
Tabela 3 - Quantidade de materiais utilizados para moldagem de dois corpos de prova cilíndricos com $50 \mathrm{~mm}$ de diâmetro $\times 100 \mathrm{~mm}$ de altura de pastas

\begin{tabular}{c|c|c|c|c|c}
\hline \multirow{2}{*}{ Pasta } & \multicolumn{5}{|c}{ Material } \\
\cline { 2 - 5 } & \multirow{2}{*}{ CP V-ARI (g) } & \multirow{2}{*}{ Sílica ativa (g) } & \multicolumn{2}{|c}{ Aditivo superplastificante } & \multirow{2}{*}{ Água (g) } \\
\cline { 4 - 5 } & & - & $\mathbf{( g )}$ & $\mathbf{( \% )}$ & 300,0 \\
\hline REF & 624,0 & 62,4 & 1,87 & 0,30 & 300,0 \\
SD & 561,6 & 62,4 & 1,87 & 0,30 & 300,0 \\
SND & 561,6 & & 0,30 & \\
\hline
\end{tabular}

Os materiais foram misturados em um misturador planetário com capacidade de 51 por 1 min na velocidade baixa (140 rpm em torno do eixo) e depois $1 \mathrm{~min}$ na velocidade alta (285 rpm em torno do eixo). Após o procedimento de mistura, foram moldados dois corpos de prova cilíndricos com $50 \mathrm{~mm}$ de diâmetro x 100 mm de altura para cada pasta. Os corpos de prova foram armazenados em câmara úmida por $24 \mathrm{~h}$, com a superfície protegida para não alterar a relação água/aglomerante, e depois em cura submersa em água saturada de cal. Após 7 e 28 dias de hidratação, os corpos de prova foram fraturados e foi realizada a paralisação da hidratação com a parte interna de cada corpo de prova. A paralisação da hidratação consistiu em colocar os fragmentos dos corpos de prova imersos em álcool etílico por $24 \mathrm{~h}$ e depois em secagem a vácuo por $10 \mathrm{~min}$ com pressão manométrica de $-500 \mathrm{mmHg}$, conforme descrito na literatura (SCRIVENER; SNELLINGS; LOTHENBACH, 2016). Após esse processo, os fragmentos foram armazenados em um recipiente hermeticamente fechado contendo sílica gel e cal sodada para evitar a hidratação e a carbonatação das amostras até a realização dos ensaios de DRX e MEV com EDS.

O ensaio de DRX foi realizado aos 7 e 28 dias de hidratação com o objetivo de avaliar o teor de $\mathrm{CH}$ das pastas. Para a realização desse ensaio, as amostras fraturadas foram moídas com o auxílio de um almofariz de ágata até todo o material passar na peneira $\mathrm{n}^{\mathrm{o}} 200$. A amostra moída foi colocada em um porta-amostra composto de uma lâmina de vidro com concavidade para adição do material através do método do pó não orientado para evitar a orientação. $\mathrm{O}$ ensaio foi realizado no difratômetro de raios X modelo Ultima IV, da marca Rigaku, operando em sistema $\theta / 2 \theta$, com velocidade de varredura de $5^{\circ} / \mathrm{min}$, de $2^{\circ}$ a $60^{\circ}$ em $2 \theta$, sob 35 $\mathrm{kV}$ e $15 \mathrm{~mA}$. A fonte de energia foi um filamento de tungstênio (cátodo) e o tubo de raios X foi de cobre

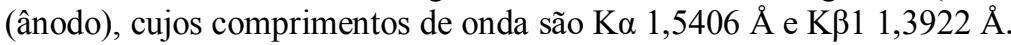

O ensaio de MEV com EDS foi realizado nas amostras fraturadas aos 28 dias de hidratação. Antes da realização do ensaio, houve recobrimento das amostras com carbono. Esse ensaio foi realizado com o objetivo de verificar a relação $\mathrm{Ca} / \mathrm{Si}$ das pastas de cimento. Na literatura, essa análise geralmente é realizada por meio de análises pontuais de EDS, verificando-se em torno de 30 pontos (ANDRADE et al., 2019; GUO et al., 2019; LEI et al., 2016). A metodologia desta pesquisa consistiu em traçar uma linha com comprimento de $200 \mu \mathrm{m}$ sobre a qual foram realizadas milhares de análises pontuais, obtendo maior confiabilidade nos resultados quando comparada com a metodologia convencional descrita anteriormente. $\mathrm{O}$ equipamento utilizado nesse ensaio foi o microscópio eletrônico de varredura modelo Jeol Quanta 450, operado em vácuo, com tensão de aceleração de elétrons de $20 \mathrm{kV}$ e detector Apollo X-SDD. As micrografias foram obtidas a partir de elétrons retroespalhados, com distância de trabalho de aproximadamente $12 \mathrm{~mm}$.

\section{Resultados e discussões}

\section{Microscopia eletrônica de varredura da sílica ativa}

Tanto a SD como a SND possuem elevado grau de aglomeração das partículas, conforme mostrado na Figura 1. Os aglomerados da SND são formados naturalmente devido à elevada finura e às tensões superficiais do material. Os aglomerados da SD, além dos fatores de aglomeração da SND citados anteriormente, são formados durante o processo de beneficiamento por aglomeração das partículas. Dessa forma, a SND possui menor tendência de aglomeração que as partículas de SD. Isso é confirmado através das imagens de MEV, nas quais a SD possui aglomerados maiores que a SND. 
Figura 1 - Imagens de MEV obtidas por meio de elétrons secundários da sílica ativa: (a) densificada (500 $\mu \mathrm{m})$; (b) densificada $(50 \mu \mathrm{m}) ;(\mathrm{c})$ não densificada $(500 \mu \mathrm{m})$; e (d) não densificada $(50 \mu \mathrm{m})$

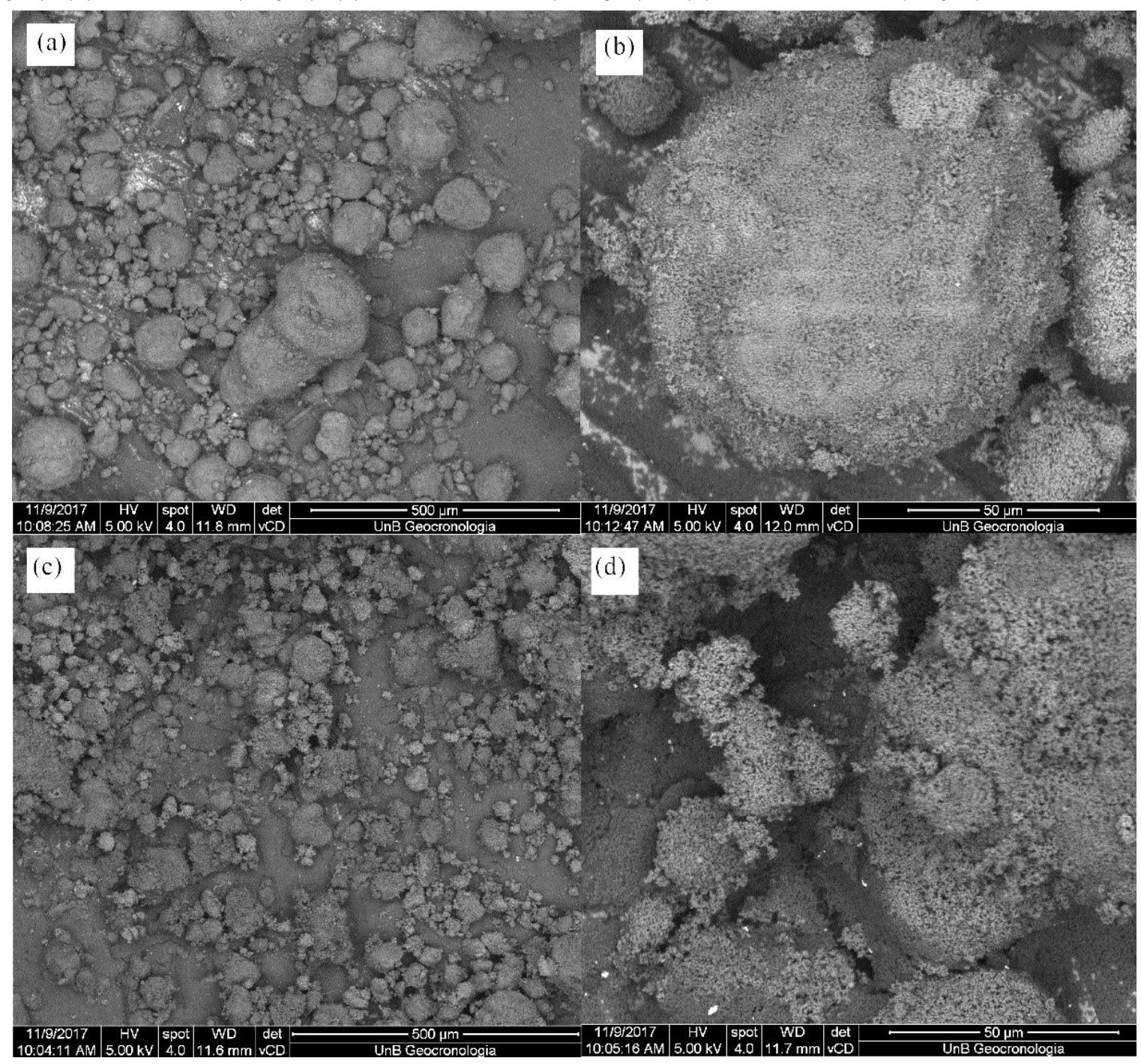

\section{Difração de raios $\mathrm{X}$ das pastas de cimento}

Os resultados de DRX das pastas aos 7 e 28 dias de hidratação são mostrados na Figura 2. Observou-se que em nenhuma das duas idades a incorporação de SA resultou no aparecimento de um novo produto, assim como observado na literatura (SENFF et al., 2010).

Observa-se na Figura 2 que as duas pastas com SA resultaram em menor teor de portlandita $\left(\mathrm{Ca}(\mathrm{OH})_{2}, \mathrm{CH}\right.$ ou hidróxido de cálcio) em comparação com a pasta REF devido à reação pozolânica e ao menor teor de cimento incorporado na mistura, corroborando com os resultados disponíveis na literatura (ANDRADE et al., 2019; GARCIA et al., 2017; GUO et al., 2019; LI et al., 2017; RÊGO et al., 2019). Além disso, houve maior consumo de $\mathrm{CH}$ da pasta SND em relação à pasta SD. Isso mostra que a sílica ativa não densificada gera uma reação pozolânica mais intensa que a sílica ativa densificada, resultando em maior consumo de $\mathrm{CH}$ para a formação de C-S-H adicional.

Devido ao menor tamanho das partículas de SND, houve maior diferença na intensidade dos picos característicos de $\mathrm{CH}$ da pasta SND em relação à pasta SD aos 7 dias do que aos 28 dias de hidratação, quando as intensidades foram próximas. Isso indica que a densificação da sílica ativa influencia no consumo de $\mathrm{CH}$ principalmente nas idades iniciais, porém não houve diferença relevante aos 28 dias de hidratação.

234 Fraga, Y. S. B.; Soares, L. M. G.; Capuzzo, V. M. S. 
Figura 2 - Difratograma de raios X das pastas REF, SD e SND aos (a) 7 dias de hidratação e (b) 28 dias de hidratação (P - portlandita, E - etringita e C - calcita)
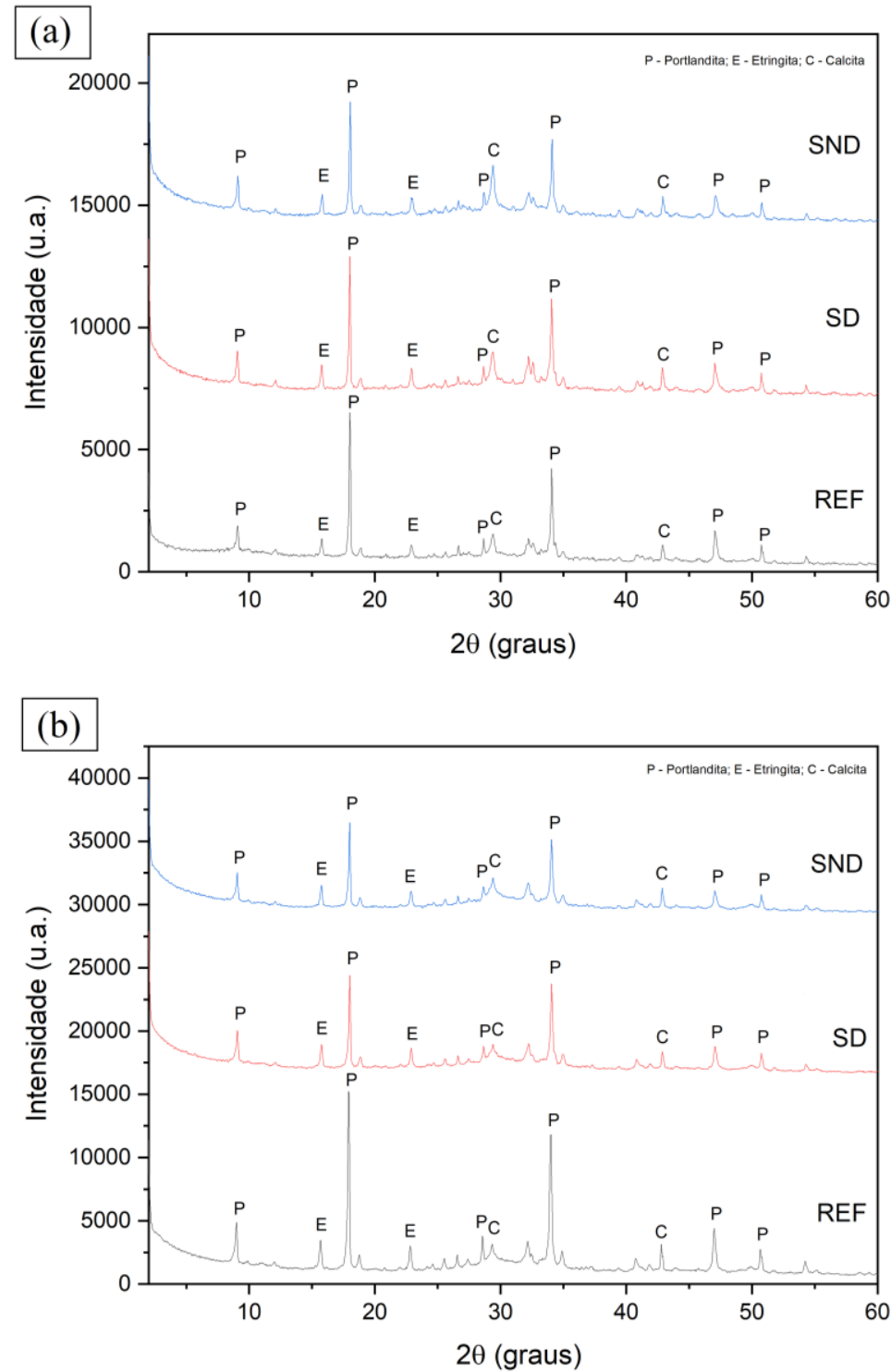

Assim como aos 7 dias de hidratação, aos 28 dias a pasta SND resultou em maior consumo do $\mathrm{CH}$ pela reação pozolânica em comparação com a SD. Esses resultados ratificam que, quanto menor o tamanho e maior a área superficial da partícula de $\mathrm{SiO}_{2}$, maior é a sua atividade pozolânica. Isso comprova que a reação pozolânica de materiais cimentícios com SA possui influência direta no grau de aglomeração da SA (FRAGA et al., 2020; SINGH et al., 2015).

As reduções das intensidades dos picos característicos de $\mathrm{CH}$ das pastas SD e SND em comparação com a pasta REF são evidentes aos 28 dias, conforme apresentado na Figura 2(b). Apesar disso, principalmente para a pasta SD, essa redução é menor aos 7 dias de hidratação, conforme a Figura 2(a). Esse comportamento indica que aos 7 dias de hidratação o menor teor de $\mathrm{CH}$ está relacionado principalmente ao menor teor de cimento Portland, enquanto aos 28 dias de hidratação essa redução é atribuída principalmente à reação pozolânica.

\section{Microscopia eletrônica de varredura das pastas de cimento}

Na Figura 3 são apresentadas as imagens de MEV obtidas através de elétrons retroespalhados das pastas de cimento aos 28 dias de hidratação, indicando uma linha com comprimento de $200 \mu \mathrm{m}$ sobre a qual foi realizada uma análise de EDS em toda a sua extensão. 
Foram destacados dois pontos na Figura 3(b) que mostraram as partículas de SD na matriz cimentícia, o que não foi observado na Figura 3(c) ao se fazer uma varredura em toda a amostra. Isso mostra que houve distribuição homogênea da SND na matriz cimentícia, favorecendo a criação de pontos de nucleação, enquanto a SD não foi completamente dispersa durante o processo de mistura das pastas.

Observa-se através da Figura 3 que o $\mathrm{Ca}$ (cor verde) pode ser do C-S-H, do $\mathrm{Ca}(\mathrm{OH})_{2}$, do $\mathrm{CaCO}_{3}$ ou do cimento anidro, pois o ensaio foi realizado na pasta de cimento, e não em determinados compostos. A identificação dos compostos pode ser realizada pela escala de cinza, sendo os tons mais claros referentes ao cimento anidro, os tons médios ao $\mathrm{Ca}(\mathrm{OH})_{2}$, os tons mais escuros ao C-S-H, e o preto indicando poros na pasta (SILVA, 2007; SHARMA et al., 2019). Na Figura 4 são mostrados os resultados do mapa de composição obtidos nos locais indicados na Figura 3.

Como a escala das intensidades varia na Figura 4, foi traçada uma linha da intensidade média do Ca e uma linha da intensidade média do Si para poder realizar a comparação das composições. A partir das linhas médias de intensidade, comparou-se visualmente a relação $\mathrm{Ca} / \mathrm{Si}$ média das três pastas analisadas.

A análise de linha possibilita fazer uma análise ao longo do comprimento da amostra, retirando a aleatoriedade da escolha dos pontos para realizar a EDS. Na Figura 4(a) observa-se a predominância de $\mathrm{CH}$ devido à baixa intensidade de Si ao longo da linha, com exceção de duas regiões, que resultaram em elevado teor de Si devido à predominância de C-S-H.

Na Figura 4(b) observa-se maior proporção de Si ao longo da linha em comparação com a pasta REF, o que pode ser atribuído à menor relação $\mathrm{Ca} / \mathrm{Si}$ e provavelmente ao aumento de $\mathrm{C}-\mathrm{S}-\mathrm{H}$. Destaca-se na Figura 4(b) que há um elevado teor de Si na região próxima ao comprimento de $200 \mu \mathrm{m}$. Através da Figura 3(b), observa-se que esse resultado é devido à presença de uma partícula de SD no fim da linha. Isso mostra que a sílica densificada é subutilizada na mistura.

$\mathrm{Na}$ Figura 4(c) foram mostradas duas regiões com maiores intensidades de Ca. Entre $0 \mu \mathrm{m}$ e $15 \mu \mathrm{m}$, esse resultado é justificado pela presença de um grão de cimento anidro, conforme se pode observar na Figura 3(c). Entre $75 \mu \mathrm{m}$ e $105 \mu \mathrm{m}$, esse resultado é justificado pela presença de CH. Nas demais regiões, foi observada maior intensidade de $\mathrm{Si}$, indicando a presença de C-S-H.

Através da análise de linhas, é possível realizar uma análise qualitativa da matriz cimentícia, recomendandose evitar traçar a linha sobre grãos de cimento anidro ou algum MCS. Ressalta-se a importância de realizar uma análise visual da imagem da pasta em conjunto com a relação $\mathrm{Ca} / \mathrm{Si}$ para evitar erros. Ao comparar a relação $\mathrm{Ca} / \mathrm{Si}$ entre as duas pastas com $\mathrm{SA}$, a pasta $\mathrm{SND}$ apresentou menor relação $\mathrm{Ca} / \mathrm{Si}$ em comparação com a pasta SD devido ao menor tamanho das partículas de SND, resultando em maior dispersão da SND na matriz cimentícia.

A redução da relação $\mathrm{Ca} / \mathrm{Si}$ da matriz cimentícia ocorre devido ao aumento no grau de polimerização do $\mathrm{C}$ S-H ao se incorporar SA na pasta de cimento (ANDRADE et al., 2019; FRAGA et al., 2020; GUO et al., 2019; ROSSEN; LOTHENBACH; SCRIVENER, 2015).

\section{Resistência à compressão axial dos microconcretos}

Os resultados de resistência à compressão dos microconcretos aos 1, 3, 7, 14 e 28 dias são mostrados na Figura 5.

Foi possível observar que o aumento da resistência à compressão do microconcreto REF foi mais acentuado até os 3 dias de hidratação devido ao maior teor de cimento na mistura e depois tendeu a estabilizar, enquanto os microconcretos SD e SND continuaram a aumentar a resistência em taxas maiores em idades posteriores. $\mathrm{O}$ microconcreto SD tendeu a estabilizar a resistência à compressão após os 14 dias de hidratação, enquanto o microconcreto SND continuou a aumentar a resistência à compressão até os 28 dias de hidratação. O resultado do índice de desempenho dos microconcretos avaliados é mostrado na Figura 6. Constatou-se que, a partir dos 7 dias, mesmo com $10 \%$ a menos de cimento Portland, os microconcretos com sílica ativa resultaram em maior índice de desempenho que o microconcreto REF.

Na Tabela 4 são mostrados os resultados da análise de variância (one-way ANOVA) da resistência à compressão axial dos microconcretos aos 1, 3, 7, 14 e 28 dias de hidratação. 
Figura 3 - Imagens de MEV obtidas a partir de elétrons retroespalhados das pastas de cimento aos 28 dias de hidratação, detalhando as linhas de análise de EDS (a) REF; (b) SD; e (c) SND

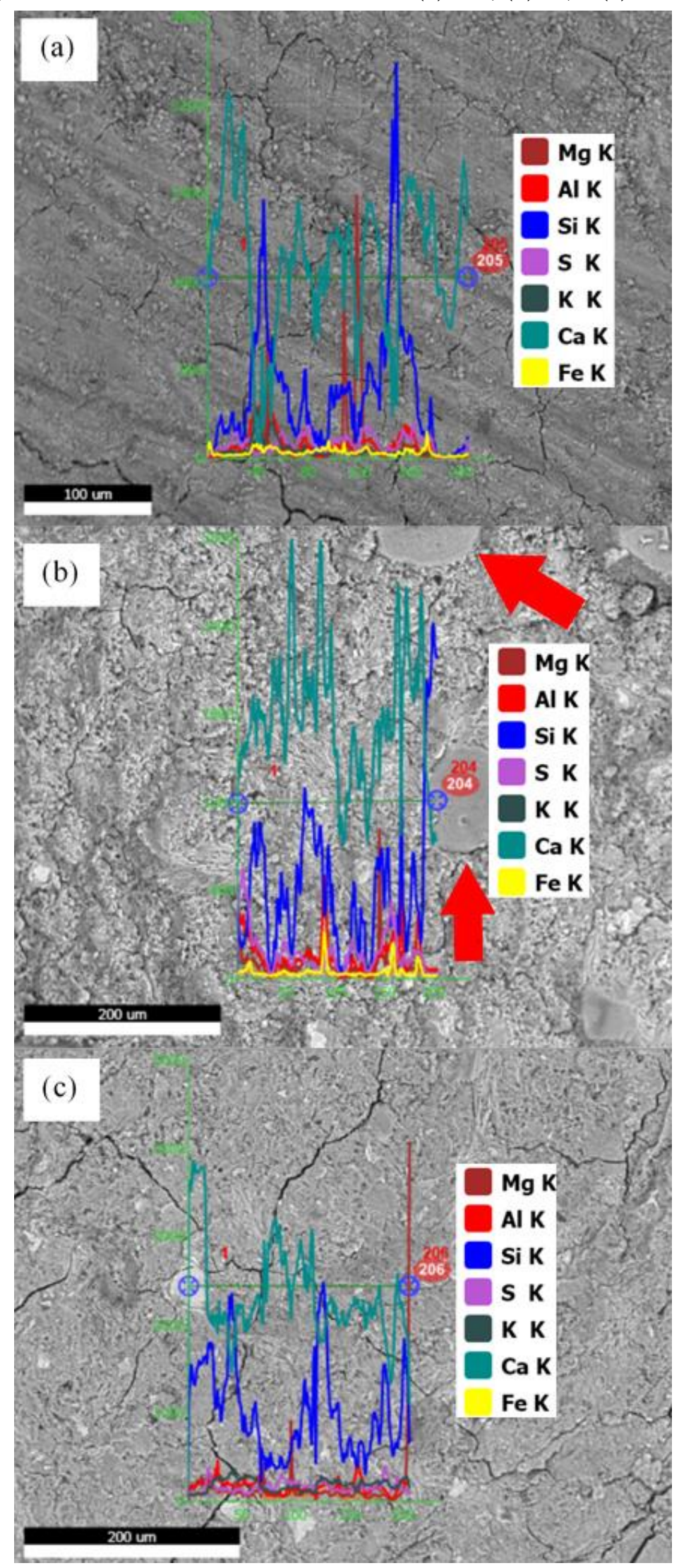


Figura 4 - Mapa de composição química com comprimento de $200 \mu \mathrm{m}$ das pastas aos 28 dias de hidratação: (a) REF com escala de intensidade entre 0 e 3.600; (b) SD com escala de intensidade entre 0 e 3.000; e (c) SND com escala de intensidade entre 0 e 4.000
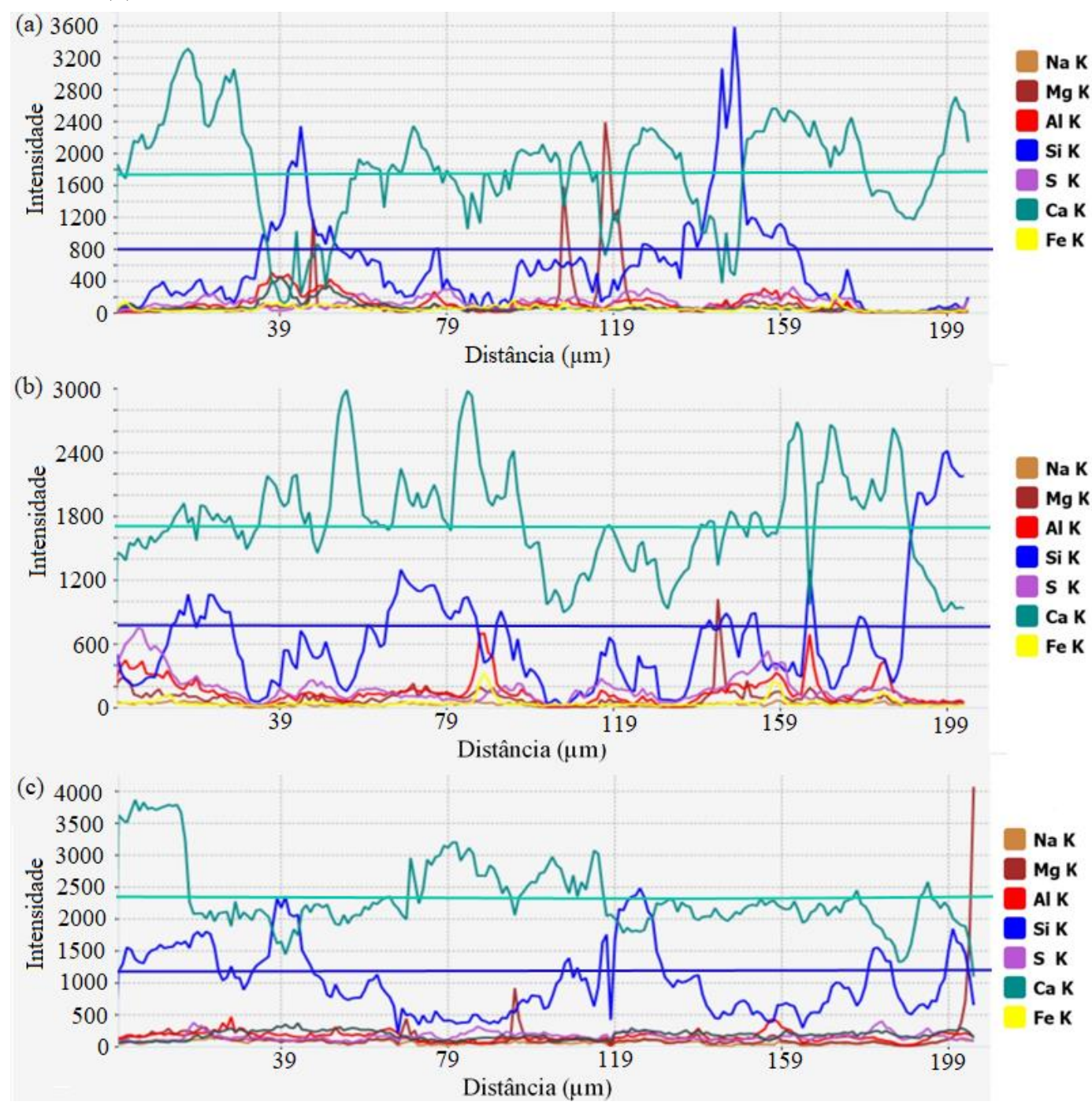

Figura 5 - Resistência à compressão axial dos microconcretos aos 1, 3, 7, 14 e 28 dias de hidratação

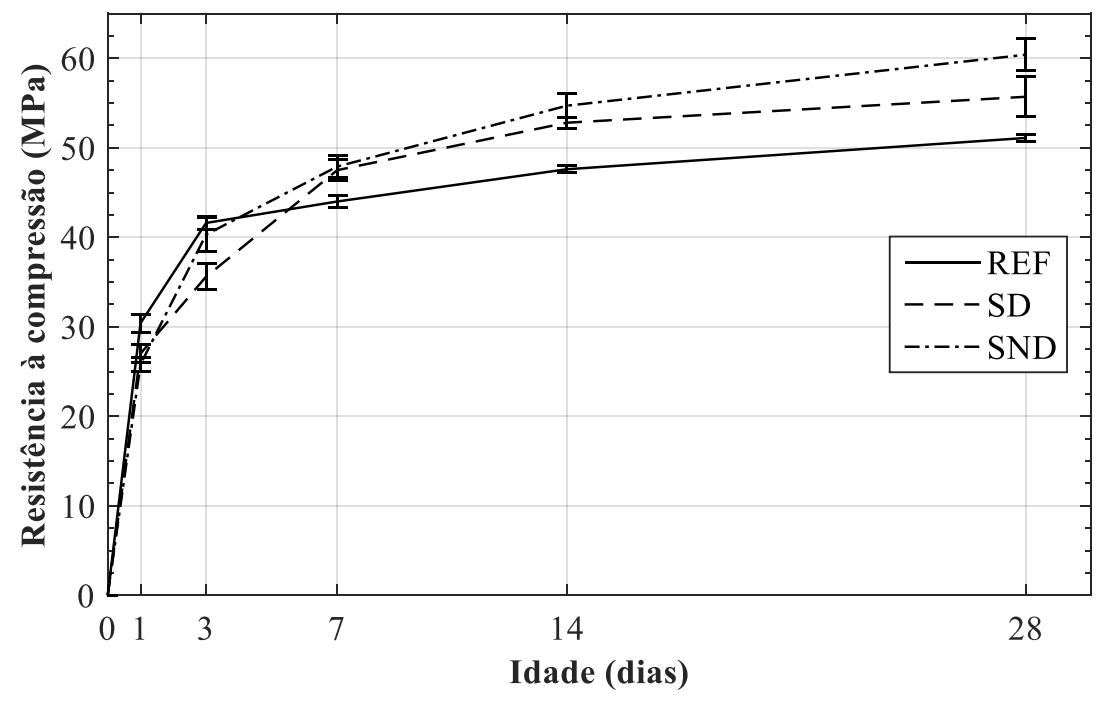


Figura 6 - Índice de desempenho da resistência à compressão axial dos microconcretos aos 1, 3, 7, 14 e 28 dias de hidratação

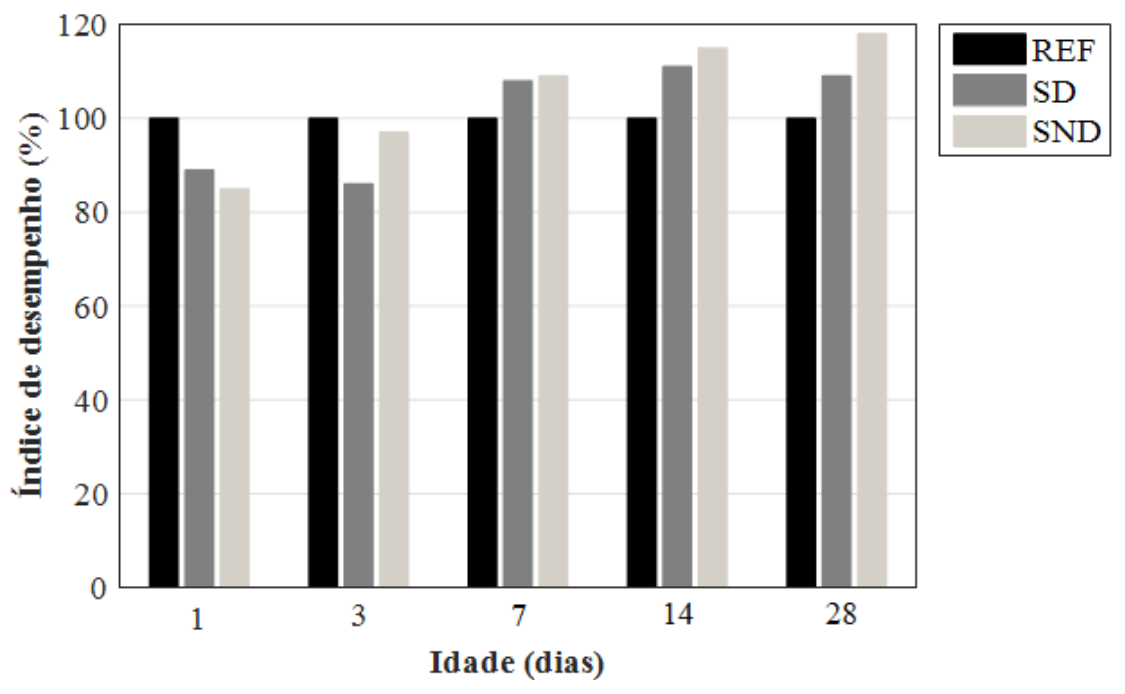

Tabela 4 - Análise de variância (one-way ANOVA) da resistência à compressão axial dos microconcretos aos $1,3,7,14$ e 28 dias de hidratação

\begin{tabular}{c|c|c|c|c|c}
\hline Idade & SQ & MQ & F & p-valor & Resultado \\
\hline 1 dia & 26,4 & 13,2 & 16,4 & 0,006391 & Significativo \\
3 dias & 64,9 & 32,5 & 12,8 & 0,004555 & Significativo \\
7 dias & 37,0 & 18,5 & 15,7 & 0,001167 & Significativo \\
14 dias & 87,4 & 43,7 & 42,2 & 0,000292 & Significativo \\
28 dias & 149,4 & 74,7 & 27,0 & 0,000514 & Significativo \\
\hline
\end{tabular}

Nota: Legenda:

$\mathrm{SQ}=$ soma dos quadrados;

$M Q$ = média dos quadrados;

$\mathrm{F}=$ parâmetro de Fisher para o teste de significância; e

p-valor $=$ probabilidade de significância.

Observou-se que, através dos resultados da análise de variância (one-way ANOVA), houve diferença significativa na resistência à compressão dos microconcretos nas idades analisadas, pois o p-valor em todas as idades foi menor que o nível de significância de 0,05 . Na Tabela 5 são mostrados os grupos homogêneos de resistência à compressão dos microconcretos aos $1,3,7,14$ e 28 dias de hidratação obtidos através do teste de Duncan, considerando a média e o desvio padrão da resistência à compressão.

A partir da Tabela 5, é possível observar que foram formados dois grupos de resistência à compressão aos 1 , 3, 7 e 14 dias de hidratação. Com 1 dia de hidratação, o microconcreto REF resultou em resistência mecânica superior aos microconcretos SD e SND, que resultaram em resistências estatisticamente iguais. Aos 3 dias de hidratação, mesmo com $10 \%$ a menos de cimento em sua composição, a microconcreto SND foi classificado no mesmo grupo de resistência à compressão que o microconcreto REF, indicando a reação pozolânica da sílica ativa não densificada entre 1 e 3 dias, enquanto o microconcreto SD resultou no menor desempenho mecânico nessa idade. Aos 7 e 14 dias de hidratação, os microconcretos com sílica ativa resultaram em desempenhos mecânicos semelhantes entre si e maiores que o microconcreto REF.

O comportamento mecânico nas idades de 1 e 3 dias pode ser justificado pela menor quantidade de cimento nos microconcretos SD e SND em comparação com o microconcreto REF. A partir dos 7 dias de hidratação, devido à reação pozolânica do $\mathrm{CH}$ proveniente do cimento Portland com a $\mathrm{SA}$, mesmo com 10\% a menos de cimento Portland, os microconcretos SD e SND resultaram em maior resistência à compressão que o microconcreto REF. Esses resultados corroboram com a literatura, na qual se observou aumento no desempenho mecânico ao utilizar 10\% de SA em conjunto com o cimento Portland devido, principalmente, à reação pozolânica da SA com o CH (ANDRADE et al., 2019; LI et al., 2017; RÊGO et al., 2019). 
Tabela 5 - Resultados do teste de Duncan para resistência à compressão dos microconcretos aos 1,3 , 7, 14 e 28 dias de hidratação

\begin{tabular}{|c|c|c|c|c|c|c|}
\hline Idade & Microconcreto & $\begin{array}{l}\text { Resistência média } \\
\text { à compressão } \\
(\mathrm{MPa})\end{array}$ & $\begin{array}{c}\text { Desvio } \\
\text { padrão } \\
\text { (MPa) }\end{array}$ & Grupo 1 & Grupo 2 & Grupo 3 \\
\hline 1 dia & $\begin{array}{c}\text { REF } \\
\text { SD } \\
\text { SND }\end{array}$ & $\begin{array}{l}30,4 \\
27,0 \\
25,8\end{array}$ & $\begin{array}{l}1,0 \\
1,0 \\
0,8\end{array}$ & $\begin{array}{l}X \\
X\end{array}$ & $\bar{X}$ & \\
\hline 3 dias & $\begin{array}{c}\text { REF } \\
\text { SD } \\
\text { SND }\end{array}$ & $\begin{array}{l}41,6 \\
35,6 \\
40,3\end{array}$ & $\begin{array}{l}0,7 \\
1,4 \\
1,9\end{array}$ & $\mathrm{X}$ & $\begin{array}{l}\mathrm{X} \\
\mathrm{X}\end{array}$ & \\
\hline 7 dias & $\begin{array}{l}\text { REF } \\
\text { SD } \\
\text { SND }\end{array}$ & $\begin{array}{l}44,0 \\
47,5 \\
47,9\end{array}$ & $\begin{array}{l}0,7 \\
1,2 \\
1,3\end{array}$ & $\mathrm{X}$ & $\begin{array}{l}X \\
X\end{array}$ & \\
\hline 14 dias & $\begin{array}{l}\text { REF } \\
\text { SD } \\
\text { SND }\end{array}$ & $\begin{array}{l}47,6 \\
52,8 \\
54,7\end{array}$ & $\begin{array}{l}0,4 \\
0,6 \\
1,3\end{array}$ & $\mathrm{X}$ & $\begin{array}{l}X \\
X\end{array}$ & \\
\hline 28 dias & $\begin{array}{l}\text { REF } \\
\text { SD } \\
\text { SND }\end{array}$ & $\begin{array}{l}51,1 \\
55,6 \\
60,4\end{array}$ & $\begin{array}{l}0,4 \\
2,2 \\
1,8\end{array}$ & $\mathrm{X}$ & $\mathrm{X}$ & $\mathrm{X}$ \\
\hline
\end{tabular}

Nota: os grupos são independentes entre as idades. Dessa forma, os grupos de uma idade não estão relacionados com os grupos das demais idades.

Aos 28 dias de hidratação, foram formados três grupos de resistência à compressão dos microconcretos. O microconcreto REF foi classificado no grupo 1, de menor resistência à compressão. O microconcreto SD foi classificado no grupo 2, com resistência à compressão superior estatisticamente ao REF, porém inferior estatisticamente ao SND. O microconcreto SND foi classificado no grupo 3, ou seja, foi o que resultou em maior resistência à compressão dentre os três microconcretos avaliados.

Esses resultados corroboram com a literatura, na qual foi observado que, quanto menor o tamanho e maior a superfície das partículas de $\mathrm{SiO}_{2}$, maior é a sua reatividade e a sua influência no refinamento da estrutura porosa, aumentando a resistência à compressão dos materiais cimentícios (FLORES et al., 2017; FRAGA et al., 2020).

Ao correlacionar os resultados de DRX, MEV e resistência à compressão, observou-se que, quanto maior o consumo de $\mathrm{CH}$ devido à reação pozolânica e menor a relação $\mathrm{Ca} / \mathrm{Si}$ da matriz cimentícia, maior a resistência à compressão dos microconcretos. Dessa forma, a utilização da SND em conjunto com o cimento Portland foi mais eficiente no consumo do $\mathrm{CH}$ e proporcionou menor relação $\mathrm{Ca} / \mathrm{Si}$ e maior resistência à compressão que a utilização da SD em conjunto com o cimento Portland.

\section{Conclusão}

A pasta referência resultou no maior teor de $\mathrm{CH}$ em comparação com as pastas contendo os dois tipos de sílica ativa aos 7 e 28 dias de hidratação. Como a reação pozolânica é lenta, houve maior influência dos dois tipos de sílica ativa no consumo de $\mathrm{CH}$ aos 28 dias de hidratação. A pasta com sílica ativa não densificada resultou no menor teor de $\mathrm{CH}$ em comparação com a pasta contendo sílica ativa densificada, comprovando que esta foi mais eficiente para promover a reação pozolânica.

A utilização dos dois tipos de sílica ativa resultou na redução da reação $\mathrm{Ca} / \mathrm{Si}$ das pastas de cimento aos 28 dias de hidratação em relação à pasta referência. Devido ao menor grau de aglomeração das partículas, a pasta com sílica ativa não densificada resultou em menor relação $\mathrm{Ca} / \mathrm{Si}$ da matriz cimentícia em comparação com a pasta com sílica ativa densificada. Através das imagens de MEV, foi possível observar grãos de SD na pasta, indicando a sua subutilização nos compósitos cimentícios.

$\mathrm{O}$ microconcreto referência resultou na maior resistência à compressão dentre os três microconcretos avaliados com 1 dia de hidratação. Após 7 dias de hidratação, os microconcretos com sílica ativa resultaram em maior resistência à compressão, mesmo com $10 \%$ a menos de cimento Portland. Ao comparar os microconcretos com os dois tipos de sílica ativa, foi observado que a sílica ativa não densificada foi mais eficaz que a sílica ativa densificada no aumento da resistência à compressão dos microconcretos.

240 Fraga, Y. S. B.; Soares, L. M. G.; Capuzzo, V. M. S. 
Diante do exposto, foi possível concluir que a sílica ativa não densificada apresentou maior atividade pozolânica que a sílica ativa densificada. Com isso, houve aumento da resistência à compressão quando incorporada a sílica ativa não densificada no microconcreto em comparação com a sílica ativa densificada. Portanto, para a produção de materiais cimentícios, recomenda-se a utilização da sílica ativa não densificada.

\section{Referências}

AÏTCIN, P. C. Concreto de alto desempenho. São Paulo: Pini, 2000.

ANDRADE, D. S. et al. Investigation of C-S-H in ternary cement pastes containing nanosilica and highlyreactive supplementary cementitious materials (SCMs): microstructure and strength. Construction and Building Materials, v. 198, p. 445-455, 2019.

ASSOCIAÇÃO BRASILEIRA DE NORMAS TÉCNICAS. NBR 7215: cimento Portland: determinação da resistência à compressão. Rio de Janeiro, 1997.

ASSOCIAÇÃO BRASILEIRA DE NORMAS TÉCNICAS. NBR 13956-1: sílica ativa para uso com cimento Portland em concreto, argamassa e pasta: parte 1: requisitos. Rio de Janeiro, 2012.

ASSOCIAÇÃO BRASILEIRA DE NORMAS TÉCNICAS. NBR 16697: cimento Portland: requisitos. Rio de Janeiro, 2018.

BAJJA, Z. et al. Influence of slurried silica fume on microstructure and tritiated water diffusivity of cement pastes. Construction and Building Materials, v. 132, p. 85-93, 2017.

BARBHUIYA, S.; CHOW, P.; DAS, A. Nanomechanical properties of cement paste containing silica fume. In: PROCEEDINGS OF INTERNATIONAL CONFERENCE ON ARCHITECTURE AND CIVIL ENGINEERING, Dubai, 2014. Proceedings [...] Dubai, 2014.

CHEN, Y.; DENG, Y; LI, M. Influence of nano-SiO2 on the consistency, setting time, early-age strength, and shrinkage of composite cement pastes. Advances in Materials Science and Engineering, p. 1-8, 2016.

CITEK, D. et al. Material properties of ultra-high performance concrete in extreme conditions. Key Engineering Materials, v. 711, p. 157-162, 2016.

DIAMOND, S.; SAHU, S.; THAULOW, N. Reaction products of densified silica fume agglomerates in concrete. Cement and Concrete Research, v. 34, n. 9, p. 1625-1632, 2004.

FLORES, Y. C. et al. Performance of Portland cement pastes containing nano-silica and different types of silica. Construction and Building Materials, v. 146, p. 524-530, 2017.

FRAGA, Y. S. B. et al. Ultrasonication and synergistic effects of silica fume and colloidal nanosilica on the C-S-H microstructure. Journal of Building Engineering, v. 32, p. 1-13, 2020.

GARCIA, D. C. S. et al. Microstructure and hardness of cement pastes with mineral admixture. Revista Matéria, v. 22, p. 1-10, 2017.

GESOGLU, M. et al. Properties of low binder ultra-high performance cementitious composites: comparison of nanosilica and microsilica. Construction and Building Materials, v. 102, p. 706-713, 2016.

GUO, Y. et al. Physically and chemically bound chlorides in hydrated cement pastes: a comparison study of the effects of silica fume and metakaolin. Journal of Materials Science, v. 54, p. 2152-1269, 2019.

JUENGER, M. C. G.; SIDDIQUE, R. Recent advances in understanding the role of supplementary cementitious materials in concrete. Cement and Concrete Research, v. 78, p. 71-80, 2015.

LEI, D. et al. A new dispersing method on silica fume and its influence on the performance of cement-based materials. Construction and Building Materials, v. 115, p. 716-726, 2016.

LI, L. G. et al. Synergistic effects of micro-silica and nano-silica on strength and microstructure of mortar. Construction and Building Materials, v. 140, p. 229-238, 2017.

LOTHENBACH, B.; SCRIVENER, K.; HOOTON, R. D. Supplementary cementitious materials. Cement and Concrete Research, v. 41, p. 1244-1256, 2011.

OERTEL, T. et al. Influence of amorphous silica on the hydration in ultra-high-performance concrete.

Cement and Concrete Research, v. 58, p. 121-130, 2014. 
RÊGO, J. H. S. et al. Effect of partial substitution of highly reactive mineral additions by nanosilica in cement pastes. Journal of Materials in Civil Engineering, v. 31, n. 1, p. 1-11, 2019.

RICHARDSON, I. G. The nature of hydration products in hardened cement pastes. Cement \& Concrete Composites, v. 22, p. 97-113, 2000.

ROSSEN, J. E.; LOTHENBACH, B.; SCRIVENER, K. L. Composition of C-S-H in pastes with increasing levels of silica fume addition. Cement and Concrete Research, v. 75, p. 14-22, 2015.

SCRIVENER, K.; SNELLINGS, R.; LOTHENBACH, B. A practical guide to microstructural analysis of cementitious materials. Boca Ratón: Taylor \& Francis Group, 2016.

SENFF, L. et al. Effect of nanosilica and microsilica on microstructure and hardened properties of cement pastes and mortars. Advances in Applied Ceramics, v. 109, p. 104-110, 2010.

SHARMA, U. et al. Effect of particle size of nanosilica on microstructure of C-S-H and its impact on mechanical strength. Cement and Concrete Composites, v. 97, p. 312-321, 2019.

SIDDIQUE, R. Utilization of silica fume in concrete: review of hardened properties. Resources, Conservation and Recycling, v. 55, n. 11, p. 923-932, 2011.

SILVA, V. M. Ação da carbonatação em vigas de concreto armado sob tensão, executadas em escala natural e reduzida. São Carlos, 2007. 279 f. Tese (Doutorado em Engenharia de Estruturas) - Universidade de São Paulo, São Carlos, 2007.

SINGH, L. P. et al. Hydration studies of cementitious material using silica nanoparticles. Journal of Advanced Concrete Technology: Materials, Structure and Environment, v. 13, p. 345-354, 2015.

ZHANG, Z.; ZHANG, B.; YAN, P. Comparative study of effect of raw and densified sílica fume in paste, mortar and concrete. Construction and Building Materials, v. 105, p. 82-93, 2016a.

ZHANG, Z.; ZHANG, B.; YAN, P. Hydration and microstructures of concrete containing raw or densified sílica fume at different curing temperatures. Construction and Building Materials, v. 121, p. 483-490, $2016 b$.

\section{Agradecimentos}

Os autores agradecem ao Conselho Nacional de Desenvolvimento Científico e Tecnológico (CNPq), à Coordenação de Aperfeiçoamento de Pessoal de Nível Superior (Capes) e à Fundação de Apoio à Pesquisa do Distrito Federal (FAPDF) (Processo $n^{\circ} 0193.001700 / 2017$ ) pelo apoio financeiro para a realização da pesquisa.

\footnotetext{
Yuri Sotero Bomfim Fraga

Departamento de Engenharia Civil e Ambiental, Faculdade de Tecnologia | Universidade de Brasília | Campus Universitário Darcy Ribeiro, Asa Norte | Brasília - DF - Brasil | CEP 70910-900 | Tel.: (61) 3107-0990 | E-mail: yurisotero.engcivil@gmail.com

Lucas Martins Gontijo Soares

Departamento de Engenharia Civil e Ambiental, Faculdade de Tecnologia | Universidade de Brasília | E-mail: lucasmartinsgs@gmail.com

Valdirene Maria Silva Capuzzo

Departamento de Engenharia Civil e Ambiental, Faculdade de Tecnologia | Universidade de Brasília | Tel.: (61) 3107-0996 | E-mail: valdirenemaria@unb.br
}

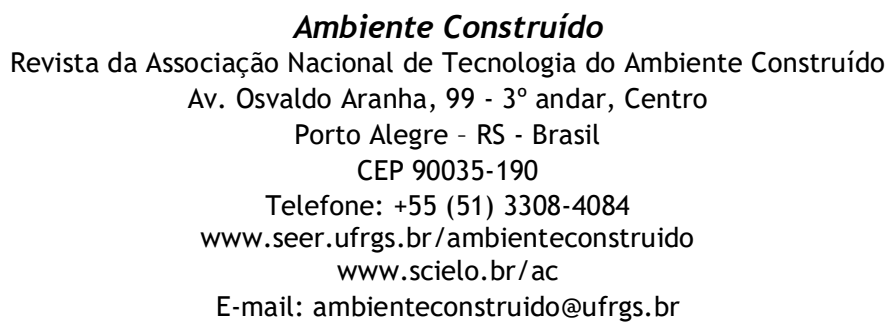

